\author{
Matthew J. McGirt \\ John A. Cowan Jr \\ Vishal Gala \\ Hugh J. Garton \\ Karin M. Muraszko \\ B. Gregory Thompson
}

\section{Surgical reversal of prolonged blindness from a metastatic neuroblastoma}

Received: 18 February 2004

Published online: 26 January 2005

C) Springer-Verlag 2005

M. J. McGirt

Department of Neurosurgery,

Johns Hopkins Medical Center,

Baltimore, MD, USA

\author{
J. A. Cowan Jr · V. Gala · H. J. Garton • \\ K. M. Muraszko \\ B. G. Thompson $(\square)$ \\ Department of Neurosurgery, \\ University of Michigan Health System, \\ 1500 East Medical Center Drive, 3552 TC, \\ Ann Arbor, MI, 48109-0338, USA \\ e-mail: gregthom@umich.edu \\ Tel.: +1-734-9367493 \\ Fax: +1-734-9369294
}

\begin{abstract}
Background: Reports of tumor-related anterior visual pathway blindness that have resolved after surgical decompression are rare. The longest reported duration of tumorrelated blindness completely reversed by optic nerve decompression is 3 days. We describe a pediatric patient with 7 days of no light perception who experienced reversal of blindness following tumor resection and optic nerve decompression. Case description: A 33-month-old boy presented with a 4-day history of no light perception. Magnetic resonance imaging revealed a mass involving the sphenoid sinus, sella turcica, and clivus with significant optic nerve involvement. Loss of light perception and complete absence of a pupillary light reflex were documented for the
\end{abstract}

next $72 \mathrm{~h}$. A sluggish pupillary light reflex was regained $24 \mathrm{~h}$ after instituting intravenous steroids. An urgent bi-frontal craniotomy and optic nerve decompression were performed 7 days after the onset of blindness. Surgical pathology revealed metastatic neuroblastoma. Eleven days after optic nerve decompression, the child was able to count fingers and recognize faces and printed book characters. Conclusion: Prolonged blindness secondary to tumor-related optic nerve compression may be reversible up to 1 week from onset in children presenting with no light perception.

Keywords Blindness . Neuroblastoma $\cdot$ Optic nerve . Surgical decompression · Visual pathways

\section{Introduction}

Acute onset of blindness due to optic nerve compression is most commonly the result of acute mass effect $[12,14]$ caused by pituitary apoplexy, cerebral artery aneurysm rupture, or trauma [15]. Although progressive tumor impingement on the optic nerve can cause visual field deficits, reports of bilateral anterior visual pathway blindness not caused by hemorrhage into a tumor are rare. To date, surgical reversal of tumor-related blindness has been limited to cases of pituitary apoplexy, meningioma, and craniopharyngioma $[1,7,8,10,11,16]$; blindness from a compressive neuroblastoma has been considered "irreversible" [2]. We report the first case of prolonged blindness due to optic nerve compression by a neuroblastoma that has been reversed by decompression.

\section{Case report}

History and examination

A 33-month-old boy with no prior medical history presented with a 4-day history of vision loss. Neurological examination revealed complete loss of light perception and an absent pupillary constriction to light in both eyes. Fundoscopic examination showed bilateral optic disk pallor. No other physical abnormalities were noted. Computed tomography (CT) scan revealed a midline mass centered at the level of the clinoid extending into the ethmoid and filling the nasopharynx with no evidence of acute hemorrhage (Fig. 1). Magnetic resonance imaging (MRI) demonstrated a $36-\mathrm{cm}^{3}$ extra-axial, heterogeneous, poorly defined midline mass involving the clivus, ethmoid and 


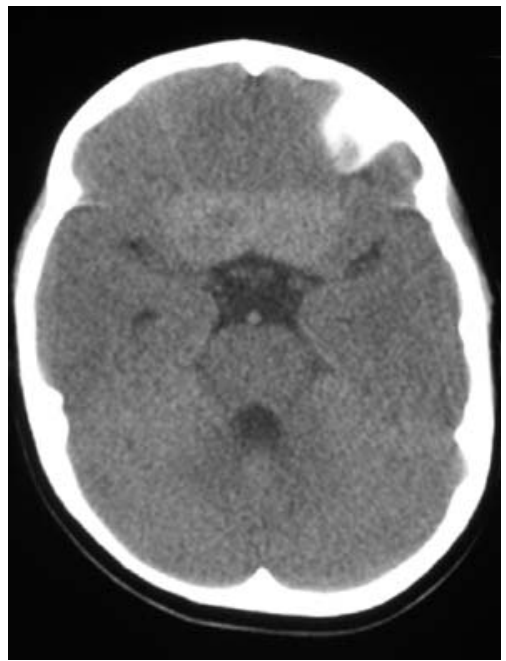

Fig. 1 Preoperative computed tomography scan of a 33-month-old male presenting with 4-day history of no light perception. A bilobed, midline, contrast-enhancing mass is centered at the level of the anterior clinoids, and extends into the ethmoid and nasopharynx with no evidence of acute hemorrhage.

sphenoid sinuses, lesser sphenoid wings, and sella turcica (Fig. 2). The optic nerves could not be identified at the foramen secondary to significant compression. A full-body MRI revealed a $3 \times 2-\mathrm{cm}$ mass involving the left adrenal gland, and multiple gadolinium-enhancing lesions within the cervical, thoracic, and lumbar vertebral bodies.

Treatment and subsequent course

An endoscopic ethmoidotomy with biopsy of the nasal and sphenoid sinus mass was performed. Microscopic examination revealed a poorly differentiated neuroblastoma. Twenty-four hours after instituting intravenous steroid therapy (7 days after the reported onset of blindness), the right pupil had a sluggish constriction to light (from 6 to $5 \mathrm{~mm}$ ); however, the patient continued to have no light perception. The patient was immediately taken to the operating room for a bilateral frontal craniotomy, tumor debulking, and bilateral optic nerve decompression. Intraoperatively, both optic nerves were encased in tumor. Postoperative imaging demonstrated a partial resection of the mass along the anterior cranial fossa with decompression and clear visualization of both optic nerves and the chiasm (Fig. 3). On postoperative day 2 , both pupils were briskly constricting (6 to $3 \mathrm{~mm}$ ) in response to light. Perception of light returned on postoperative day 5. After 1 week, the patient could count fingers $2 \mathrm{ft}$ away and tracked objects with his eyes. After receiving a 3-day course of vincristine sulfate, adriamycin, and cytoxan therapy, the patient was discharged home 11 days after surgery with the ability to recognize television and book characters.

\section{Intervention}

Following a bicoronal skin incision, an osteoplastic bifrontal craniotomy and a bilateral orbital bar osteotomy were performed. The pterional boney resection was extended posteriorly toward the orbital apex with Leksell rongeurs. After opening the dura, self-retaining retractors were used to gently elevate the frontal lobe, and the operating microscope was inserted to expose the opticocarotid triangle. The dura was abnormally elevated along the sphenoid wing by the tumor, which initially obscured the proximal intracranial carotid artery and the optic nerve. Additional exposure was achieved by opening the proximal sylvian fissure. Tumor growth within the dura had expanded and softened the anterior clinoid. An H-shaped incision into the dura overlying this area revealed firm, vascular, and somewhat friable tissue. Using sharp dissection under high microscopic magnification, we excised the clinoidal dura and removed the tumor overlying the distal dural ring, expanding the clinoid. The tumor also had eroded the optic canal, so we used a high-speed drill
Fig. 2 Sagittal T1-weighted magnetic resonance images of a heterogeneous and poorly-defined mass centered at a level of the clinoid. a Pregadolinium. b Postgadolinium.
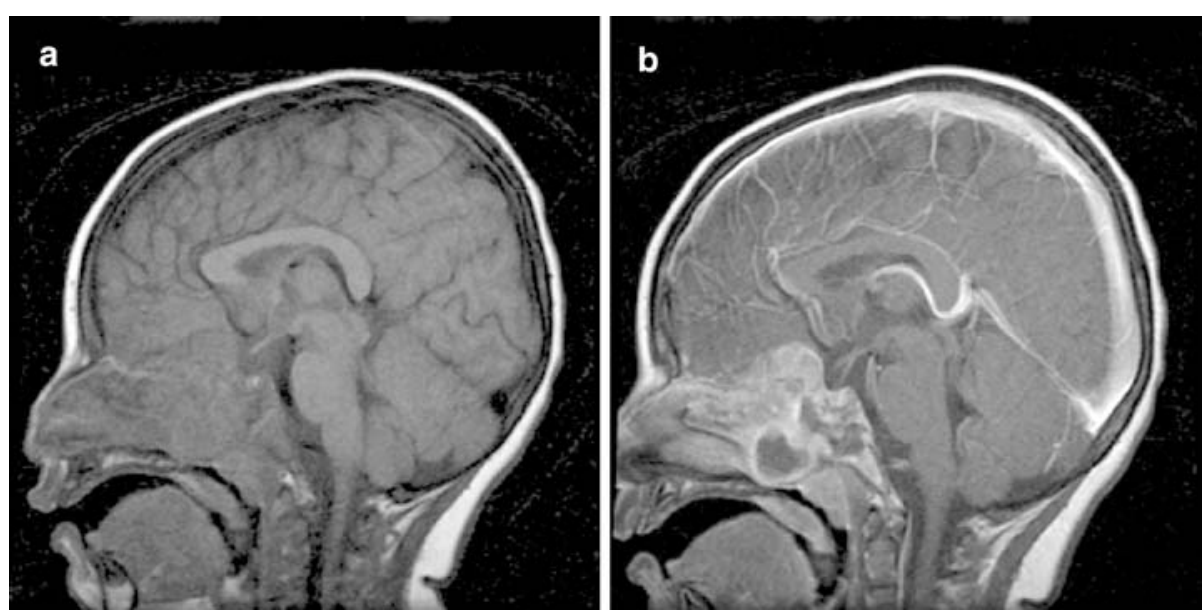
Fig. 3 Postoperative computed tomography scans revealing serial sections (a-d, inferior to superior) through the anterior skull base. The patient underwent a partial resection of the tumor with decompression of both optic nerves.
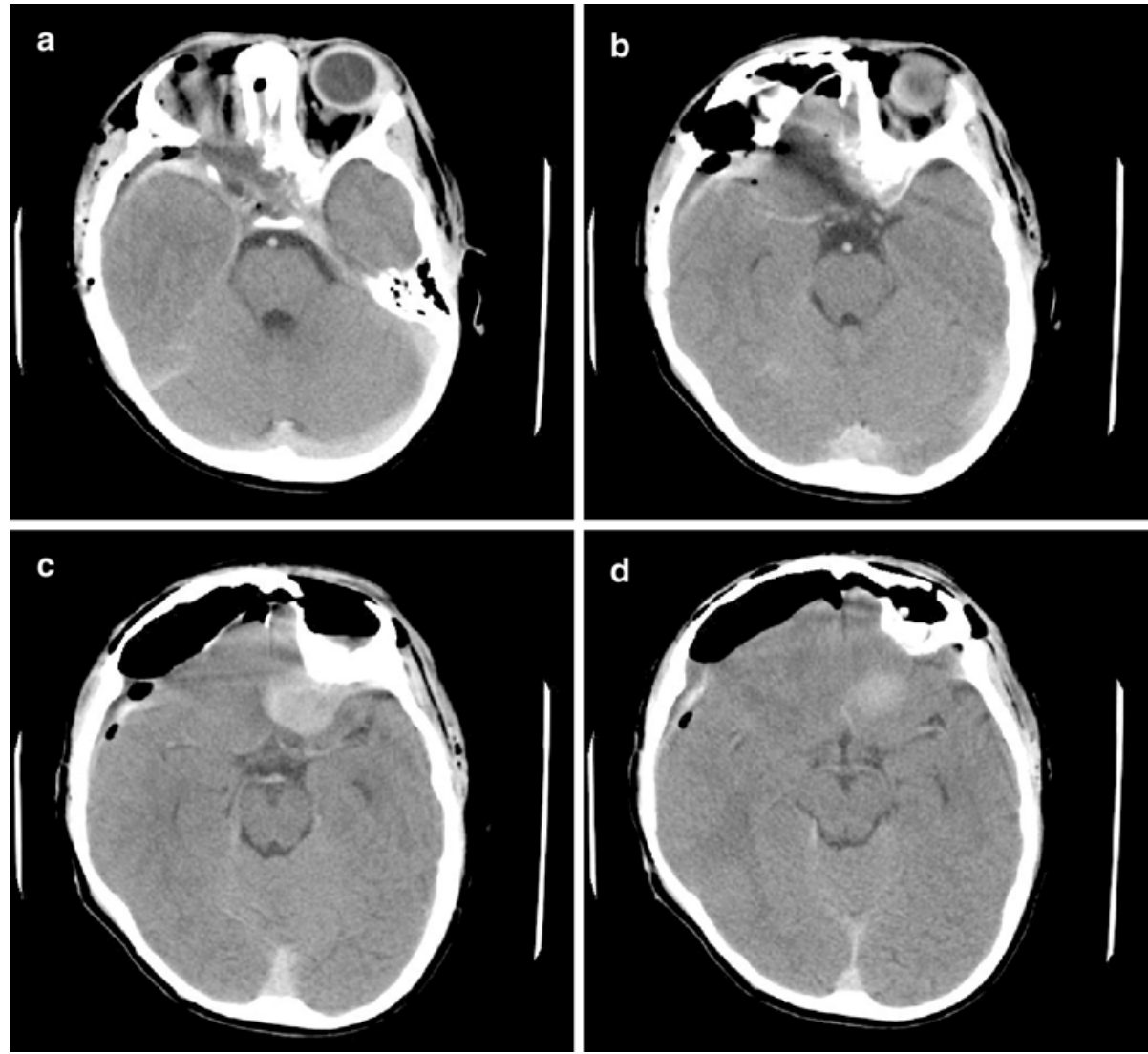

with a 3-mm diamond drill bit to remove residual bits of the eroded optic nerve canal and further release the optic nerve on the right side. The falciform ligament was also opened over the optic nerve, which had been bowed and stretched by the tumor. We opened the dural ring of the optic nerve and dissected the surrounding tissues and associated tumor until the nerve was entirely exposed to its entrance into periorbital tissues.

The tumor resection was then carried out medially into what would ordinarily be the region of the ethmoid sinuses but which, in this patient, had been entirely displaced by tumor. Additional layers of bone were removed until the left optic nerve was identified and decompressed in like fashion.

Closure was accomplished utilizing a dural graft where the tumor had been excised, and by rotating in a vascularized pericranial graft overlying the region of the ethmoids (to preclude omission of any open sinus areas). The orbital bar and the osteoplastic craniotomy flap were then reattached with absorbable microplates and screws.

\section{Discussion}

Anterior visual pathway compression blindness has previously been described as reversible within $72 \mathrm{~h}$ of onset $[1,5,7,8,10,11,16-18]$. Al-Wahhabi et al. reported reversal of 72-h blindness following optic nerve decompression in a 3-year-old with a craniopharyngioma [1]. Tajima et al. described reversal of blindness following decompression $48 \mathrm{~h}$ after loss of vision in a patient with a large unruptured anterior cerebral artery aneurysm [18]. Optic nerve decompression in the setting of prolonged blindness has been less encouraging, however. Maurer et al. reported no improvement in vision following optic nerve decompression in 7 patients experiencing blindness for 4 or more days due to a meningioma [11]. Other authors have also reported failure to reverse meningioma-induced blindness with optic nerve decompression when loss of light perception was present for more than 1 week $[8,10$, 16]. Striph et al. reported the only case of reversed blindness greater than $72 \mathrm{~h}$ after onset, accomplished by decompressing a giant anterior communicating artery aneurysm 5 days after loss of light perception [17].

The underlying pathophysiology of optic nerve compression is poorly understood. Ischemic mechanisms are the most widely accepted cause of optic nerve dysfunction following compression [6]. In our case, the 10-day duration of recovery as well as the near-complete return of vision would argue against vascular compromise caused by arterial insufficiency [3] or venous stasis [9]. CliffordJones et al. demonstrated that optic nerve demyelination occurs within 1 week of compression, with remyelination beginning after 5 weeks of compression [4]. It is unclear 
how the more rapid and near-complete recovery made in our patient could occur by this more chronic mechanism. Stretching of the axonal membranes or deformation of myelin with displacement of the nodes of Ranvier [13] allows for a reversible conduction block in subacute compression of the optic nerve and may explain the phenomenon observed in our patient.

Despite complete blindness from optic nerve compression, our patient demonstrates that if some discernible pu- pillary light reflex is present, surgical decompression can result in very good recovery of vision, even when delayed for longer than $72 \mathrm{~h}$.

Acknowledgements The authors would like to acknowledge the editorial support of Holly Wagner, Department of Neurosurgery, University of Michigan Health System, in the creation of this manuscript.

\section{References}

1. Al-Wahhabi B, Choudhury AR, AlMoutaery KR et al (1993) Giant craniopharyngioma with blindness reversed by surgery. Childs Nerv Syst 9:292-294

2. Belgaumi AF, Kauffman WM, Jenkins JJ et al (1997) Blindness in children with neuroblastoma. Cancer 80:19972004

3. Bergland RM, Ray BS, Torack RM (1968) Anatomical variations in the pituitary gland and adjacent structures in 225 human autopsy cases. J Neurosurg 28:93-99

4. Clifford-Jones RE, Landon DN, McDonald WI (1980) Remyelination during optic nerve compression. J Neurol Sci 46:239-243

5. Dutton JJ, Tse DT, Anderson RL (1983) Compressive optic neuropathy following use of intracranial oxidized cellulose hemostat. Ophthalmic Surg 14:487-490
6. Frisen L, Sjostrand J, Norrsell K et al (1976) Cyclic compression of the intracranial optic nerve: patterns of visual failure and recovery. J Neurol Neurosurg Psychiatry 39:1109-1113

7. Gorman RL, Ahlstrom B, KobrinMerritts D (1986) Acute blindness. Am J Emerg Med 4:459-463

8. Gregorius FK, Hepler RS, Stern WE (1975) Loss and recovery of vision with suprasellar meningiomas. J Neurosurg 42:69-75

9. Hoyt W (1970) Correlative functional anatomy of the optic chiasm. 1969. Clin Neurosurg 17:189-208

10. Jallu A, Kanaan I, Rahm B et al (1996) Suprasellar meningioma and blindness: a unique experience in Saudi Arabia. Surg Neurol 45:320-323

11. Maurer J, Hinni M, Mann W et al (1999) Optic nerve decompression in trauma and tumor patients. Eur Arch Otorhinolaryngol 256:341-345

12. McFadzean RM, Doyle D, Rampling R et al (1991) Pituitary apoplexy and its effect on vision. Neurosurgery 29:669675
13. Ochoa J, Danta G, Fowler T et al (1971) Nature of nerve lesion caused by pneumatic tourniquet. Nature 233:265-266

14. Parent AD (1990) Visual recovery after blindness from pituitary apoplexy. Can J Neurol Sci 17:88-91

15. Robinson JL (1972) Sudden blindness with pituitary tumors. Report of three cases. J Neurosurg 36:83-85

16. Rosenstein J, Symon L (1984) Surgical management of suprasellar meningioma. II. Prognosis for visual function following craniotomy. J Neurosurg 61:642-648

17. Striph GG, Slamovis TL, Burde RM (1984) Visual recovery following prolonged amaurosis due to compressive optic neuropathy. J Clin Neuroophthalmol 4:189-195

18. Tajima A, Ito M, Ishii M (1993) Complete recovery from monocular blindness caused by aneurysmal compression to optic nerve: report of two cases. Neurol Med Chir 33:19-23 